UCRL-JC-130674

PREPRINT

\title{
Kinetics and Thermodynamic Behavior of Carbon Clusters under High Pressure and High Temperature
}

\author{
R.H. Ree \\ N.W. Winter \\ J.N. Glosli
}

This paper was prepared for submittal to the 36th European High Pressure Research Group Meeting on Molecular and Low Dimensional Systems under Pressure

Catalina, Italy

September 7-11, 1998

May 4, 1998

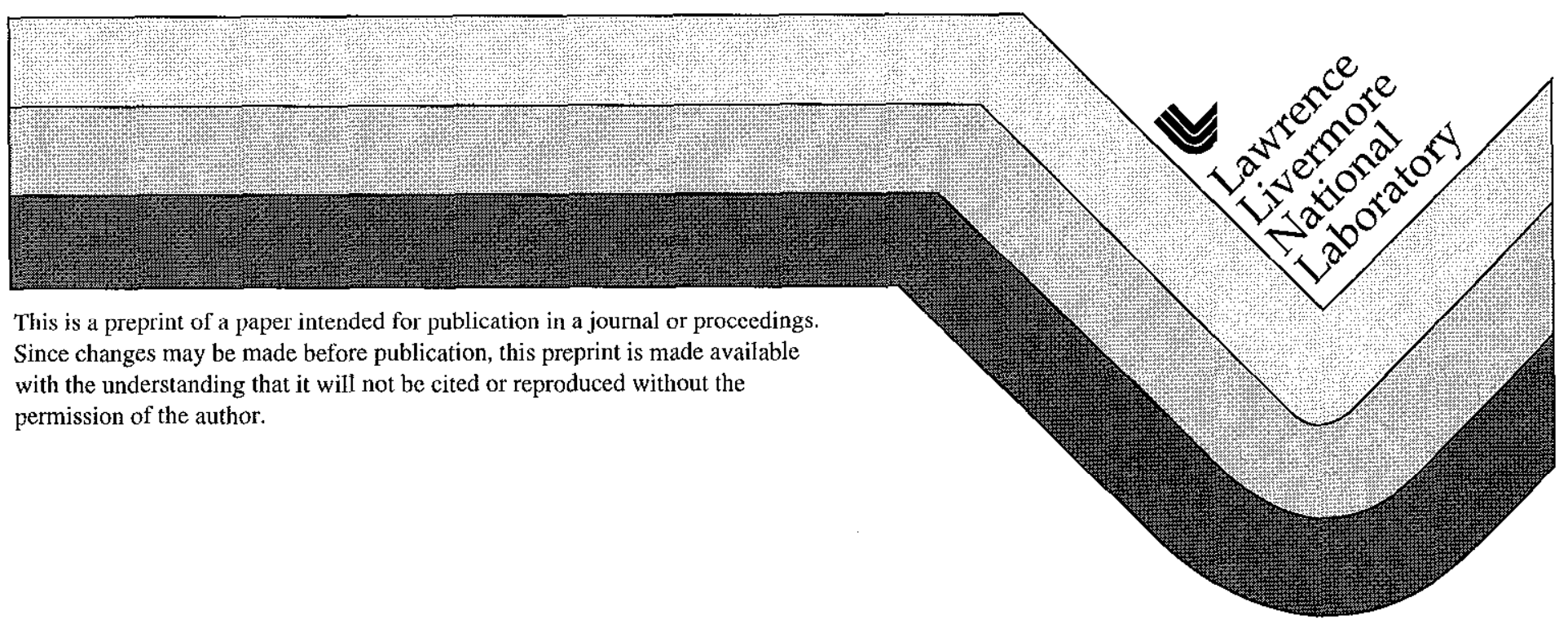




\section{DISCLAIMER}

This document was prepared as an account of work sponsored by an agency of the United States Government. Neither the United States Government nor the University of California nor any of their employees, makes any warranty, express or implied, or assumes any legal liability or responsibility for the accuracy, completeness, or usefulness of any information, apparatus, product, or process disclosed, or represents that its use would not infringe privately owned rights. Reference herein to any specific commercial product, process, or service by trade name, trademark, manufacturer, or otherwise, does not necessarily constitute or imply its endorsement, recommendation, or favoring by the United States Government or the University of California. The views and opinions of authors expressed herein do not necessarily state or reflect those of the United States Government or the University of California, and shall not be used for advertising or product endorsement purposes. 
Kinetics and thermodynamic behavior of carbon clusters under high pressure and high
temperature

\author{
F.H. Ree, N.W. Winter, J.N. Glosli \\ Lawrence Livermore National Laboratory, P.O. Box 808, Livermore, CA 94550, USA \\ ree1@llnl.gov, winter3@llnl.gov, glosli1@llnl.gov
}

\begin{abstract}
Physical processes that govern the growth kinetics of carbon clusters at high pressure and high temperature are: (a) thermodynamics and structural ( $s p^{2}$-to-s $s p^{3}$ bonding) changes and (b) cluster diffusion. Our study on item (a) deals with $a b$ initio and semi-empirical quantum mechanical calculations to examine effects of cluster size on the relative stability of graphite and diamond clusters and the energy barrier between the two. We have also made molecular dynamics simulations using the Brenner bond order potential. Results show that the melting line of diamond based on the Brenner potential is reasonable and that the liquid structure changes from mostly $s p$ bonded carbon chains to mostly $s p^{3}$-bonding over a relatively narrow density interval. Our study on item (b) uses the time-dependent cluster size distribution function obtained from the relevant Smoluchowski equations. The resulting surface contribution to the Gibbs free energy of carbon clusters was implemented in a thermochemical code. These results show that the behavior of carbon-rich explosives is sensitive to the metastability of graphitic carbon clusters during the diffusion of carbon clustering processes.
\end{abstract}

A Japanese experiment ${ }^{1}$ has shown that glassy carbon transforms to graphite below $3,000 \mathrm{~K}$ if it is held at $9 \mathrm{GPa}$ for 5 minutes and is quenched subsequently. Carbon should have been in the diamond phase under this condition. Obviously, the 5 minute- heating period was not long enough for amorphous carbon to transform to diamond, since the same experiment at higher temperatures produced diamond crystals. Our analyses ${ }^{2}$ of high explosives with high carbon contents also supports the Japanese result. Namely, carbon microclusters in detonation products will occur in the graphitic phase within the pressure-temperature range where the diamond phase is thermodynamically stable. Both experiments point out that the size of clusters is important to the stability of the diamond or graphite phase and that the temperature and pressure are two important variables controlling the growth rate of cluster sizes.

The first part of this paper addresses an issue on why carbon atoms prefer to transform to an intermediate (graphite) phase prior to the diamond formation. Its understanding is crucial to the reliable prediction of high explosives detonation. Amlöf and Lüthi ${ }^{3}$ earlier carried out $a b i n i$ tio quantum mechanical total energy calculations on the phase stability of graphite and diamond clusters. These calculations are computationally costly. Calculations involving larger carbon clusters, as required in the present case, can be done more efficiently by using semiempirical molecular orbital methods. We have done calculations with semiempirical molecular orbital and the firstprinciples density function theory (DFT) methods for clusters with $s p^{2}$ or $s p^{3}$ bonding constrained to have the bulk graphite or diamond structure. The singly occupied surface orbitals were capped with $H$ atoms in order to enforce the bulk $C$ atom hybridization at the surface. The system sizes used range up to $C_{286} H_{144}$ for diamond clusters and $\mathrm{C}_{294} \mathrm{H}_{42}$ for graphite clusters. We used the calculated bond energies, van der Waals interactions, and the experimental heats of formation of bulk graphite and diamond to predict that the graphite phase becomes energetically favored over the diamond structure for 15,000 to $100,000 C$ atoms. But its precise value is sensitive to level of approximations. Calculations using the PM3 and AM1 parameterizations and the DFT give roughly similar results.

Next, the barrier height as a function of cluster size was determined and compared to previous local density calculations of the activation energy for the bulk graphite to diamond phase transition. We have carried out DFT, $a b$ initio and semiempirical unrestricted Hartree-Fock calculations of the energy by the linear synchronous transit (LST) approximation which assumes all atoms at intermediate states to move synchronously and linearly along a reaction coordinate from the initial $\left(100 \% s p^{2}\right)$ to the $\left.100 \% s p^{3}\right)$ structure. The structures of the initial and final structures were determined by molecular mechanics optimization. The remaining structures are linear interpolations 
between these endpoints. The resulting barrier height is in agreement with the LDA results of bulk activation energies. ${ }^{4}$ Considering the differences in the methods being compared, the agreement in the shape and location of the transformation barrier is excellent.

The second part of this paper deals with diffusion kinetics and thermodynamic aspects of carbon clusters. On the thermodynamic side, we explored the phase diagram of carbon at high temperature and pressure via molecular dynamics using the bond order potential developed by Brenner. 5 This potential allows for realistic breaking and formation of bonds and reproduces correct bond strengths for many bonding possibilities found in hydrocarbon systems, including correct structures for all the common hybridization states $\left(s p, s p^{2}\right.$ and $\left.s p^{3}\right)$ of carbon under ambient condition and allowance for the breaking and formation of covalent bonds. The particular features examined are the diamond melting line and the local structure of the liquid phase.

The resulting melting line agrees remarkably well with experiment near the graphite- diamondliquid triple point. The slope of the melting line is positive, in agreement with high pressure sound speed experiment. The original Brenner potential represents only the covalent-bonding with a short-range cutoff. We need to include the nonbonded term to simulate sheet-sheet interactions which hold two-dimensional graphite structures together. This effort is in progress.

Liquid carbon has never been "seen" under a controlled laboratory environment. A recent flash-heating experiment by Togaya ${ }^{8}$ sugests an intriguing possibility ${ }^{6,7}$ that the liquid carbon may have two phases separated by a first-order phase change. In light of this we have been exploring the structure of the liquid phase by computer simulations with the Brenner potential. The local structural change with density appears to occur over a relatively small density interval. Though it alone is not an evidence for a phase transformation, it is suggestive of one.

In addition to the diamond-graphite transition kinetics, we need to consider the coagulation kinetics of carbon clusters by diffusion. The present model uses Smoluchowski equations to describe a change in concentration $c_{k}$ of $k$ atom clusters with time; ${ }^{9}$

$$
d c_{k} / d t=\sum_{i+j=k} k_{i j} c_{i} c_{j}-2 c_{k} \sum_{j} k_{j k} c_{j},
$$

where $k_{i j} \equiv 4 \pi\left(D_{i}+D_{j}\right) R_{i j}\left(D_{i}=\right.$ diffusion constant of a clusters with $i$ atoms; $R_{i j}=$ collision diameter between clusters of sizes $i$ and $j$ ). Using an approximate but reliable solution $c_{k}$, the timedependent surface energy correction $\langle\Delta E\rangle$ to the bulk carbon energy can be obtained by averaging $\Delta E_{k} \equiv E(k=\infty)-E(k)$ over $c_{k}$.

We have implemented $\langle\Delta E\rangle$ in a multiphase multi-component chemical equilibrium code to account for the surface correction to the Gibbs free energy of diamond and graphite clusters at a given time. An application of the code has shown that a difference between theory and experiment in the detonation velocity of TNT at some initial density is attributable to metastable processes involving nanometer size clusters of carbon formed during detonation.

\section{Acknowledgments}

This work was done under the auspices of the US DOE at the Lawrence Livermore National Laboratory under contract No. W-7405-ENG-48.

\section{References}

1. S. Hirano, K. Shimono, and S. Naka, J. Mat. Sci. 17, 1856 (1982)

2. M. van Thiel and F.H. Ree, J. Appl. Phys. 62, 1761 (1987).

3. J. Amlöf and H.P. Lüthi, ACS Symp. Series: Supercomputer Research in Chemistry and Chemical Engineering, eds. K.F. Jensen and D.G. Truhlar (ACS, WA, 1987).

4. S. Fahy, S.G. Louie, and M.L. Cohen, Phys. Rev. B 35, 7623 (1987).

5. D. Brenner, Phys. Rev. B 42, 9458 (1990).

6. A. Feraz and M.H. March, Phys. Chem. Liq. 8, 289 (1979).

7. M. van Thiel and F.H. Ree, Phys. Rev. B 48, 3591 (1993).

8. M. Togaya, Phys. Rev. Lett. 79, 2474 (1997).

9. M.S. Shaw and J.D. Johnson, J. Appl. Phys. 62, 2080 (1987). 